\title{
ANTICANCER ACTIVITY OF POLYMERIC NANOPARTICLES CONTAINING ETOPOSIDE USING ALBINO RATS AS EXPERIMENTAL ANIMAL MODEL
}

\author{
AYYAPPAN T ${ }^{1,2 *}$, SHANMUGAM $S^{1}$ \\ ${ }^{1}$ Department of Pharmaceutics, Adhiparasakthi College of Pharmacy, Melmaruvathur - 603 319, Kanchipuram, Tamil Nadu, India. \\ ${ }^{2}$ Research Scholar, The Tamil Nadu Dr. M.G.R. Medical University, Chennai - 600 032, Tamil Nadu, India. Email: tayyaps79@gmail.com
}

Received: 26 June 2018, Revised and Accepted: 31 July 2018

\section{ABSTRACT}

Objective: Etoposide is a potent anticancer drug and a major limiting factor that hinders therapeutic use as its high levels of systemic circulation often associated with various off-target effects, particularly cardiotoxicity. The present study focuses on the evaluation of the anticancer activity of optimized etoposide nanoparticles compared with marketed etoposide formulation in experimental animal models.

Methods: Albino rats (180-200 g) were used for the study. The anticancer effect of the formulated etoposide nanoparticles was evaluated by induction of hepatocellular carcinoma (HCC). The animals housed in cages and kept on a $12 \mathrm{~h} \mathrm{light/dark} \mathrm{cycle.} \mathrm{Following} \mathrm{the} \mathrm{acclimatization} \mathrm{for} 1$ week, HCC will be induced in rats by adding $100 \mathrm{mg} / \mathrm{L}$ of diethylnitrosamine (DENA) in drinking water for 8 weeks. Drug treatment (etoposide [9 mg/kg] in $0.5 \%$ carboxymethyl cellulose; p.o.) can be started at the end of 1 week after cancer induction and dose of 9 mg/kg of etoposide and lyophilized nanoparticles will be administered through oral route on 1, 7, 14, 21, and 28 days. Nanoformulations were evaluated in healthy rats, and anticancer activity was studied in DENA treated rats.

Results: The results showed that the polymeric nanoparticles containing etoposide was screened for its anticancer activity by counting the liver nodules of rats with hematoxylin and eosin stain when comparing control and etoposide-treated group.

Conclusion: The results of the study concluded that in rats treated with nanoformulations, the numbers of liver nodules were found to be significantly reduced.

Keywords: Etoposide, Anticancer, Hepatocellular carcinoma, Diethylnitrosamine.

(C) 2018 The Authors. Published by Innovare Academic Sciences Pvt Ltd. This is an open access article under the CC BY license (http://creativecommons. org/licenses/by/4. 0/) DOI: http://dx.doi.org/10.22159/ajpcr.2018.v11i12.28127

\section{INTRODUCTION}

Cancer is a major public health problem in the world. There were 14.1 million new cancer cases and 8.2 million cancer deaths in 2012 worldwide. If these rates do not change, the global cancer burden is expected to nearly double to 21.4 million cases and 13.5 million deaths by 2030 . Whereas the breast cancer is the most common cancer among women population in worldwide, with nearly 1.7 million new cases diagnosed in 2012 (the second most common cancer). This survey represents about $12 \%$ of all new cancer cases and $25 \%$ of all cancers in women. Cancer is the second leading cause of death worldwide and was responsible for 8.8 million deaths in 2015. As per the WHO, nearly 1 in 6 deaths is due to cancer.

A typical example of topoisomerase inhibitors is etoposide, and it is the first line of chemotherapeutic agents that are used in the treatment of many types of cancer. The mechanism of action of etoposide by forming a ternary complex with topoisomerase II and DNA, causing DNA breaks, and cell death [1]. In addition to this, there are many side effects related to the drug [2-4], the administration of etoposide is rate limited by its low solubility in aqueous solutions [5,6]. Therefore, finding an effective approach to facilitate the transport of drugs and to improve the bioavailability of therapeutics is necessary.

The drug candidate etoposide has variable oral bioavailability and ranging from $24 \%$ to $74 \%$ and has terminal half-life of $1.5 \mathrm{~h}$ by intravenous route and $0.44 \mathrm{~h}$ by the oral route. The conventional oral therapy has the drawback of low bioavailability, and parenteral therapy causes inconvenience and pain to the patients as it has to be given through a continuous IV infusion over 24-34 h. Hence, the objective of the present study was made for anticancer activity of etoposide polymeric nanoparticles in comparison with pure and marketed formulation using albino rats as an animal model.

\section{MATERIALS AND METHODS}

Materials

Etoposide was a gift sample from Biocon Limited, Bengaluru, India; Eudragit ${ }^{\circledR}$ EPO and HPMC K-15 were gifts from Cipla Pharmaceuticals, Mumbai, India. Pluronic ${ }^{\circledR}$ F-68 gifted from Alembic Pharmaceuticals, Mumbai, India. High-performance liquid chromatography grade of methanol, water, and acetonitrile was purchased from Qualigens fine chemicals Mumbai, India. All other reagents and chemicals used in this study were of analytical grade.

\section{Preparation of Eudragit EPO based nanoparticle suspension} Nanoparticles suspensions were prepared by nanoprecipitation method. Dissolved the drug $(50 \mathrm{mg})$ and specific amount of Eudragit ${ }^{\Theta_{-}}$ EPO $(0.45 \%)$ in $15 \mathrm{ml}$ of methanol. The organic solution quickly injected to $40 \mathrm{ml}$ aqueous solution containing Pluronic ${ }^{\circledR}$ F-68 under stirring at $2000 \mathrm{rpm}$. Stirring was continued for $2 \mathrm{~h}$ at $40^{\circ} \mathrm{C}$ for complete evaporation of methanol. The volume was adjusted up to $40 \mathrm{ml}$ with an aqueous solution of $200 \mathrm{mg}$ of HPMC K-15 to obtain a nanoparticle suspension. The optimized nanoparticles suspension was lyophilized at $-42^{\circ} \mathrm{C}$ for $72 \mathrm{~h}$ and which also redispersed in water to get aqueous nanoparticles suspension [7].

In previous work, $3^{2}$ factorial designs were used to optimize the process parameters such as polymer concentration and stabilizer concentration. Two dependent variable's drug content, entrapment efficiency was measured as responses. Mathematical equations and response surface plots were used to relate the dependent and independent 
variables. The particle size of optimised formulation was found to be $131.4 \pm 0.057 \mathrm{~nm}$, entrapment efficiency of about $94.28 \pm 0.198 \%$ and drug content of $88.36 \pm 0.075 \%$. The observed responses were in close agreement with the predicted values of the optimized process. The prepared nanoparticle was characterized by Fourier transform infrared spectroscopy, X-ray diffraction, and morphological studies, in vitro drug release studies, kinetic modeling, and stability studies. The prepared nanoparticle was showed good sustained release of drug up to $24 \mathrm{~h}$ [8]. Based on in vitro parameters, the optimized subjected to anticancer activity studies and its comparison with the marketed formulation.

\section{Evaluation of anticancer activity}

\section{Experimental animals}

Animals (albino rats) were allowed standard pellet food and water ad libitum.

Animals housed in colony cages under standard laboratory conditions $\left(12: 12 \mathrm{~h}\right.$ light and dark cycles, temperature maintained at $25 \pm 2^{\circ} \mathrm{C}$, and relative humidity $55 \pm 10 \%$ ). Animals were allowed to acclimate for 7 days to the laboratory conditions before the conduct of experiments. An experimental protocol approved by the Institutional Animal Ethical Committee (APCP/IAEC/409/01) before starting the research work. Experiments were performed in accordance with the current guidelines of CPCSEA.

Based on the results of in vitro studies, the optimized nanoparticles were chosen for this study. The anticancer activity was assessed using albino rats.

\section{Induction of hepatocellular carcinoma (HCC)}

Male rats (weight: 180-200 g) will be housed in cages and kept on a $12 \mathrm{~h}$ light/dark cycle. Following the acclimatization for 1 week, HCC will be induced in rats by adding $100 \mathrm{mg} / \mathrm{l}$ of diethylnitrosamine (DENA) in drinking water for 8 weeks [9-11].

\section{Treatment}

Anticancer experiments will be carried out using 6 rats (cancer-induced as above) in each group. The rats will be divided into three groups and maintained for 1 week.

Group I - Control to receive only $0.5 \% \mathrm{w} / \mathrm{v}$ sodium carboxymethyl cellulose (CMC); p.o.

Group II - To receive etoposide ( $9 \mathrm{mg} / \mathrm{kg})$ in $0.5 \%$ CMC; p.o.

Group III - To receive optimized nanoparticles containing equivalent amount of etoposide ( $9 \mathrm{mg} / \mathrm{kg}$ ) in $0.5 \%$ CMC; p.o.

Drug treatment can be started at the end of 1 week after cancer induction, and dose of $9 \mathrm{mg} / \mathrm{kg}$ of etoposide and lyophilized nanoparticles will be administered through oral route on $1,7,14,21$, and 28 days.

\section{Analysis}

During treatment, weight of animals will be monitored. Following treatment completion on day 28 , the animals will be sacrificed under anesthetic ether. The liver can be removed, and lobes will be separated. Tumor nodules on the surface of each lobe measuring $3 \mathrm{~mm}$ in diameter (measured with a digital caliper) will be counted, and the difference in the number of nodules in the three experimental groups is needed to be statistically evaluated. A second evaluation of tumor growth will be performed after the lobes are fixed in $10 \%$ formalin, which makes neoplastic nodules more evident. The upper and lower surfaces of each fixed lobe, together with a millimeter graded bar will be photographed for the digital enlargements of the photos. Since lumps smaller than $2 \mathrm{~mm}$ could not be identified with much certainty as nodules, this is not counted. In livers displaying a multi-nodular surface, identifiable nodules will be counted to a maximum of 50 , since the total number could not be reliably assessed. The antitumor effect of the drugs and nanoparticles was estimated by comparing the number of animals with more than 50 tumor masses in each of the different experimental groups. Samples of all the lobes of the liver will be fixed in $10 \%$ formalin embedded in paraffin and were routinely stained with hematoxylin and eosin $(\mathrm{H}$ and $\mathrm{E})$

\section{Statistical analysis}

Results are expressed as a mean \pm standard error of the mean $(n=6)$. The statistical analysis of data was done using one-way analysis of variance, followed by Dunnett's t-test. Probability level $<0.05$ was considered statistically significant.

\section{RESULTS AND DISCUSSION}

\section{Histological analysis of treated rats}

Hepatic cellular carcinoma is induced by oral administration of DENA. The results of the study showed the significant formation of HCC as evidenced by the formation of nodules on liver tissue and also from the cancerous cellular growth profile in liver histochemical sections (Fig. 1). When the rats were treated with etoposide, the nodule formation was found relatively reduced with some cancerous growth still evident in liver sections (Fig. 1). The rats who were treated with etoposide showed that the many numbers of the nodules have disappeared and suggesting the maximum inhibition of HCC, which is further confirmed by histochemical analysis of liver tissue sections showed with the control group. These observations suggest that the polymeric nanoparticle formulations of etoposide are highly efficacious compared to etoposide. Mean body weight (MBW) was measured for each group animals. DENA induced HCC rat shows an increase in MBW was found to be $204.66 \mathrm{~g} \pm$ 0.816 . When treated with etoposide, its MBW is decreased significantly indicating the severe toxic effects exerted by the drug $(193.83 \mathrm{~g} \pm 1.169$, while in the case of treatment with etoposide MBW of animals was found significantly restored $(180.33 \mathrm{~g} \pm 0.516)$ with a slight decrease in weight due to inhibition of cancer growth. Thus, nanoparticle encapsulation of etoposide has reduced the etoposide-related weight loss establishing its reduced off-target effects when delivered with polymeric nanoparticles.

Fig. 1 shows the treatment of HCC. HCC was induced by adding $100 \mathrm{mg} / \mathrm{L}$ of DENA in drinking water for 8 weeks. After 1 week of the gap from the DENA treatment, animals received $9 \mathrm{mg} / \mathrm{g}$ of drug equivalent of Etoposide through oral administration on 1, 7, 14, 21, and 28 days. Panel A shows untreated liver with a maximum number of nodules which were reduced to some extent when treated with Etoposide (panel B). HCC was significantly reduced when treated with etoposide nanoparticles (Panel C), which is indicated by the reduction in the number of nodules. It is further analyzed by $\mathrm{H}$ and $\mathrm{E}$ analysis of liver nodules along with treatments.

\section{CONCLUSIONS}

The present investigation concludes that the anticancer activity of etoposide polymeric nanoparticles was found to be statistically significant in comparison with pure and marketed formulation using albino rats as an animal model.

\section{ACKNOWLEDGMENTS}

The authors are very much thankful to his holiness Arulthiru Bangaru Adigalar, President, with respect to Thirumathi V. Lakshmi Bangaru Adigalar, Vice President, for providing entire facilities for the research work. Authors are also thankful to Prof. Dr. T. Vetrichelvan, Principal, Adhiparasakthi College of Pharmacy, Melmaruvathur, and those who helped us in direct or indirect way to carry out this study successfully.

\section{AUTHOR'S CONTRIBUTIONS}

Authors contributed to the design and implementation of the research, to the analysis of the results, and to the writing of the manuscript. 


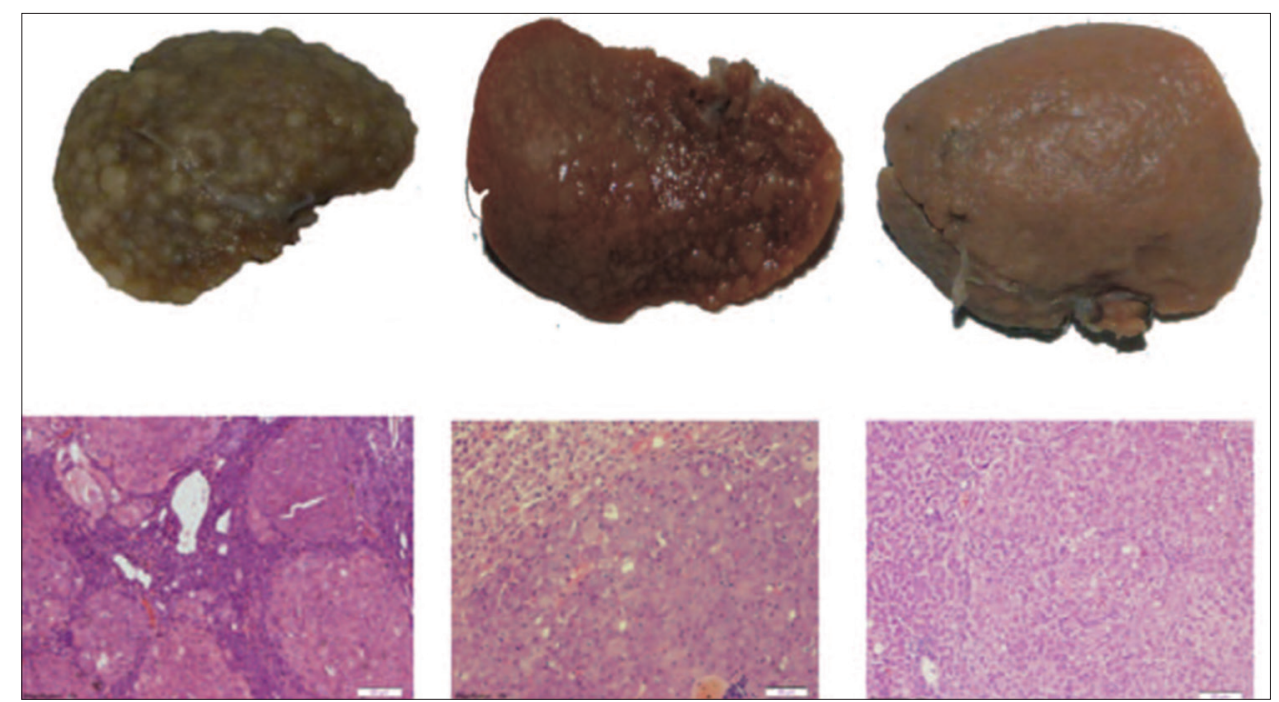

A. Control

B. Etoposide

C. Polymeric nanoparticles

Fig. 1: Anticancer activity (Panel A) shows untreated liver with maximum number of nodules which were reduced to some extent when treated with etoposide (panel B). HCC was significantly reduced when treated with etoposide nanoparticles (Panel C), which is indicated by the reduction in the number of nodules. It is further analyzed by hematoxylin and eosin analysis of liver nodules along with treatments

\section{CONFLICTS OF INTEREST}

The author declares that they have no conflicts of interest.

\section{REFERENCES}

1. Montecucco A, Biamonti G. Cellular response to etoposide treatment. Cancer Lett 2007;252:9-18.

2. Ezoe S. Secondary leukemia associated with the anti-cancer agent, etoposide, a topoisomerase II inhibitor. Int J Environ Res Public Health 2012;9:2444-53.

3. McLeod HL, Evans WE. Clinical pharmacokinetics and pharmacodynamics of epipodophyllotoxins. Cancer Surv 1993;17:253-68.

4. Rodman JH, Murry DJ, Madden T, Santana VM. Altered etoposide pharmacokinetics and time to engraftment in pediatric patients undergoing autologous bone marrow transplantation. J Clin Oncol 1994;12:2390-7.

5. Hande KR. Etoposide pharmacology. Semin Oncol 1992;19:3-9.

6. Joel SP, Shah R, Slevin ML. Etoposide dosage and pharmacodynamics.
Cancer Chemother Pharmacol 1994;34 Suppl: S69-75.

7. Chellampillai B, Pawar AP. Improved bioavailability of orally administered andrographolide from $\mathrm{pH}$-sensitive nanoparticles. Eur J Drug Metab Pharmacokinet 2011;35:123-9.

8. Thiyagarajan A, Saravanabhavan S, Thangarasu V. Preparation and biopharmaceutical evaluation of novel polymeric nanoparticles containing etoposide for targeting the cancer cells. Turk J Pharm Sci 2018. (In Press)

9. Fonseca C, Simões S, Gaspar R. Paclitaxel-loaded PLGA nanoparticles: Preparation, physicochemical characterization and in vitro anti-tumoral activity. J Control Release 2002;83:273-86.

10. Sangeetha N, Kumaraguru AK. Antitumor effects and characterization of biosynthesized iron oxide nanoparticles using seaweeds of Gulf of Mannar. Int J Pharm Pharm Sci 2015;7:469-76.

11. Preethi R, Padma PR. Anticancer activity of silver nanobioconjugates synthesised from Piper betle leaves extract and its active compound eugenol. Int J Pharm Pharm Sci 2019;8:201-5. 